\title{
Application of Fuzzy Optimization Method in Decision-Making for Personnel Selection
}

\author{
Masuma Mammadova, Zarifa Jabrayilova \\ Institute of Information Technology of Azerbaijan National Academy of Science, Baku, Azerbaijan \\ Email: depart15@iit.ab.az
}

Received 11 September 2014; revised 5 October 2014; accepted 18 October 2014

Copyright @ 2014 by authors and Scientific Research Publishing Inc.

This work is licensed under the Creative Commons Attribution International License (CC BY). http://creativecommons.org/licenses/by/4.0/

(c) (7) Open Access

\begin{abstract}
The paper describes the necessity of application of intelligent technologies to support decisions of more objective problems in human resource management. In this paper, we describe the methodology for personnel selection problem for the vacancy with regard to the importance and nonequivalence of numerous indicators characterizing the alternatives. The specific features of the selection problem are highlighted, immersing the problem into a fuzzy environment. A fuzzy multicriterial model of the personnel selection problem is proposed. A technique of order preference by similarity to ideal solition (TOPSIS), was applied for evaluation and regulation of alternatives. This technique is based on criteria of qualitative character, which are hierarchically structured by multiple experts to intellectually support decisions made in personnel selection problem. Using TOPSIS method and generated criteria system an experiment was conducted for evaluation of the candidates during solution of hiring problems. The obtained and reviewed results were compared with results obtained using in reality.
\end{abstract}

\section{Keywords}

Support Decision, Human Resource Management, Personnel Selection Problem, Fuzzy Multicriterial Model, Criteria Coefficients, Fuzzy Number, TOPSIS Method

\section{Introduction}

In the transition to knowledge-based economy, ensuring effective performance and competitiveness of the organization (enterprises, companies, firms, etc.) requires increased attention to the personnel, i.e. human factor. Employees of the organizations are considered as the main strategic resource, ensuring its performance and achievements of its objectives. According to this concept, the staff becomes one of the main resources of the organization and the necessary funds must be invested to ensure its proper management and optimal conditions for its development [1]. 
The concept basis of personnel management constitutes an increasing role of the worker's individuality, his knowledge of motivational attitudes, and his ability to shape and direct them in accordance with the challenges facing the organization. Intelligent capital occupies a special position among other assets and requires specific approaches to the management perspective [2]. Evaluation of intelligent capital of the organization is needed to determine its effectiveness and growth factors, as well as to make decisions on the advisability of investment in this resource.

Objectives of human resource management (HRM) are the basis of personnel policy. The correct solution to these problems, making objective and transparent decisions on HRM allows the organization to achieve its global goals [3] [4]. In general, today, an effective HRM becomes the strategy of the company. In this case, the funds invested in the development of human resources, transform into an investment, not expenditure [5]. The changes, occurred in the labor market, require major changes in the relationship with employees, in the policy of their recruitment, retention and motivation. In this regard, human resource management at the professional level has become a strong modern means used in HR. Fundamentally new attitude towards the personnel as valuable resource of the organization actualizes the importance of developing new conceptual approaches and technologies for HRM. Therefore, in recent years, computer technology is increasingly used for the HRM problem solutions.

Thus, to make more objective decisions regarding personnel planning, selection, recruitment, adaptation, firing, promotion, development, training and motivation of personnel the decision-maker (DM) must evaluate and take into account the information in each case, that characterizes the applicant, his interests, potential impacts and results. Essential factor for the quality of personnel management is its assessment using competencies. The problems solved in the field of HRM are complex and varied. They are united by the fact that the finite number of evaluated objects is used as the raw data, and these objects are characterized by a set of diverse features, i.e. these tasks are multicriterial, and many factors should be taken into account, many influences, preferences, interests and consequences, characterizing alternatives should be evaluated [6]-[8].

Volume, quantitative and qualitative nature, complexity and contradictions of the information flow to be reached to the decision-makers, as well as the need to address the interrelationship of numerous factors, dynamic situation created difficulties in decision-making on human resource management. To overcome these difficulties, and consequently, more effective HRM of the organization the application of intelligent decision support technologies seems appropriate [7]-[9].

The following problems belong to the HRM problems that are most frequently met in practice [4] [10]: selection of applicant on a vacant position; compliance of workers to requirements of a workplace, a position; formation of a personnel reserve and planning of vocational advancement, career; selection of people on key positions in operation of business; awarding, compensation of employees etc.

In this paper, we describe the methodology for personnel selection problem for the vacancy with regard to the importance and nonequivalence of numerous indicators characterizing the alternatives (candidates applying for the position).

\section{Personnel Selection Problem}

Personnel selection often acts the most important role for controlling the human source and quality in HRM [4] [11] [12]. Effective employee selection is a critical component of a successful organization. Personnel selection is the process of collecting and evaluating information about individuals and choosing those who match the qualifications needed to perform a predefined job in the best way [13]. This process plays a determining role in HRM and is crucial to the success of an organization.

References [14] and [15] reviewed the personnel selection studies and found that the several main factors including change in organizations, change in work, change in personnel, change in the society, change of laws, and change in marketing have influenced personnel selection. In literature, there are a number of studies which use heuristic methods for employee selection. A fuzzy MCDM framework based on the concepts of ideal and anti-ideal solutions for the most appropriate candidate is presented in [16]. Also, a fuzzy number ranking method by metric distance for personnel selection problem was proposed in [17] and a personnel selection system based on fuzzy AHP was developed in [18].

In addition, researchers used fuzzy technique for order preference by similarity to ideal solition (TOPSIS) based on the veto threshold for ranking job applicants [19]-[22]. 
Recently, owing to the advancements in information technology, researchers have developed decision support systems and expert systems to improve the outcomes of HRM [23] [24].

A model to design an expert system for effective selection and appointment of the job applicants is developed in [25]. The applications of expert system or decision support systems on personnel selection and recruitment are increasing [11] [12] [26]. In this paper, the description of fuzzy decision-making support method in the solution of employment problems is given, and comparison of the results obtained by this method application and the results obtained by real use of approach are presented hereunder.

Therefore, the goal of personnel selection is applying a valid and effective method to reduce the risks of hiring an unsuitable employee, and increase the opportunities to find an eligible employee who can enhance the productivity of the organization [4] [27]. In other words, businesses find eligible employees meet the requirements of organization and occupation from mass job applicants through effective personnel selection methods.

The technique for the personnel selection proposed by the authors in this paper has the following advantages: 1) allows the use of both qualitative and quantitative data; 2) removes the limit on the number of criteria and the number of experts; 3) takes into account the hierarchical structure of criteria. The main advantage of the article is to carry out the step calculation and allowing the comparison of experimental results with real data.

\section{Conceptual Model of the Personnel Selection Problem}

\subsection{The Specific Features of the Personnel Selection Problem}

The problem of personnel selection for the position is classified as semi-structured tasks, which is traditionally reduced to decision-making [7] [9] [19]-[24]. The attitude of the decision-maker and preferences (experience, knowledge and intuition) of the experts play an important role in the implementation of such tasks. Intelligence support policy of choice (selection of experts), in this case, is defined by a specific manager-DM, experts involved in the evaluation process of alternatives for set of attributes forming the level of satisfaction of alternatives criteria and preference relations for each of them, and the estimating problem of the applicants for the position can be reduced to the adjustment of alternatives in fuzzy initial information.

Before shifting to the methods of candidates' selection, it is important to formalize requirements for the position or workplace of the future employee, based on the development strategy of the organization and characteristics of its corporate culture. For selecting the employee, it is necessary to determine the presence or absence of a candidate's competence, which is needed for the effective performance, i.e. a set of knowledge, skills, abilities, social and personal characteristics and behavior of employees, defined with the objectives of the organization and set for specific situation. Approach based on competences allows one to link a whole HRM: in recruitment, career planning, assessment of performance and development in the promising coming years [2]. For selecting the candidates, their competence is assessed and compared with the "portrait of an ideal employee", conveyed by a set of corporate performance at a given workplace [4] [7] [19]-[24] [28] [29]. Note that the competence of a person is characterized by a number of factors and indicators, and depending on the fields of professional activity, profession and profile of the organization, these figures have different relative weights of importance [7] [19]-[22] [30] [31].

Recently, a new trend in the selection of personnel has been observed, which is expressed in individual requirements of the employers for applicants for a certain position, which involves an assessment of the latter one from the standpoint of obligation, desirability and the lack of demand characterizing the indicators with respect to the proposed position. Hence, the figure, which is mandatory according to the preference of one employer for the purposes and needs of another one, may be desirable or even unnecessary [32].

Accordingly, as semistructured, the problem of personnel selection is characterized by the following features:

- multifactorial and multicriteriality;

- criteria and indicators of qualitative and quantitative nature;

- the need to consider the views in the evaluation process;

- hierarchy rate criteria characterizing evaluated object, expressed in the fact that each top-level individual criterion is based on the aggregation of partial criteria.

These features "immerse" the task of hiring into a fuzzy environment, i.e. into the "medium-Zade", and cause decision making on the selection of the most suitable candidate for the position in poorly defined fuzzy situation [33] [34].

Thus, an evaluation model referring to fuzzy formalism for development of an intellectual system supporting 
decision making person for realization and reflecting expert knowledge must be proposed (desirability, obligation and unimportance of criteria indicators).

So, the following must be known for solution of evaluation issue in solution of staff management issues requiring intelligent support:

- Set of evaluated alternatives: $X=\left\{x_{1}, x_{2}, \cdots, x_{n}\right\}=\left\{x_{i}, i=\overline{1, n}\right\}$;

- Set of criteria characterizing alternatives: $K=\left\{K_{1}, K_{2}, \cdots, K_{m}\right\}=\left\{K_{j}, j=\overline{1, m}\right\}$;

- Set of evaluable indicators characterizing each criteria: $K_{j}=\left\{k_{j 1}, k_{j 2}, \cdots, k_{j T}\right\}=\left\{k_{j t}, t=\overline{1, T}\right\}$;

- Value range of each evaluable indicator- $Y$;

- Expert group participating in evaluation (decision making process) $-E$;

- Relations in $X, K$ and $E$ sets- $P$;

- Linguistic expressions reflecting the level of relevance and relation of alternatives to criteria indicators $-L$.

- Relative relations in same-group indicators and criteria sets- $W$.

Listed components of selection are united in below relative-set model: $M s=(X, K, Y, E, P, L, W)$.

Solution of evaluation and selection issue based on this model requires development of a relevant method, which refers to solution methods of multi-criteria issues using fuzzy mathematical formalism for this purpose [33] [35].

Current article reviews the application issue of TOPSIS method for evaluation and regulation of alternatives (selected, regulated) evaluated for intellectual support of decisions made in personnel selection issues based on hierarchically structured criteria of qualitative character by multiple experts.

\subsection{TOPSIS Method}

For the realization of selection issue, the level of relevance and relation of alternatives to criteria indicators, based on conversion of linguistic expressions of quality of our natural language to a fuzzy number (triangle or trapeze) based on proximity to an ideal solution and remoteness from an extremely bad solution traditional are carried out using TOPSIS method allowing discovery of the best solution and ranging of alternatives. In the reviewed case, trapeze fuzzy number has been used.

Definition 1. Trapeze fuzzy number membership function is a fuzzy set depicted as below (Figure 1).

Trapeze fuzzy number is indicated as $\left(n_{1}, n_{2}, n_{3}, n_{4}\right)$ quadruple and here $n_{1}, n_{2}, n_{3}, n_{4}$-are real numbers. Fuzzification of trapeze fuzzy number is defined as below:

$$
\mu(x)= \begin{cases}0, & x<n_{1}, \\ \frac{x-n_{1}}{n_{2}-n_{1}}, & n_{1} \leq x \leq n_{2}, \\ 1, & n_{2} \leq x \leq n_{3}, \\ \frac{x-n_{4}}{n_{3}-n_{4}}, & n_{3} \leq x \leq n_{4} . \\ 0, & x>n_{4} .\end{cases}
$$

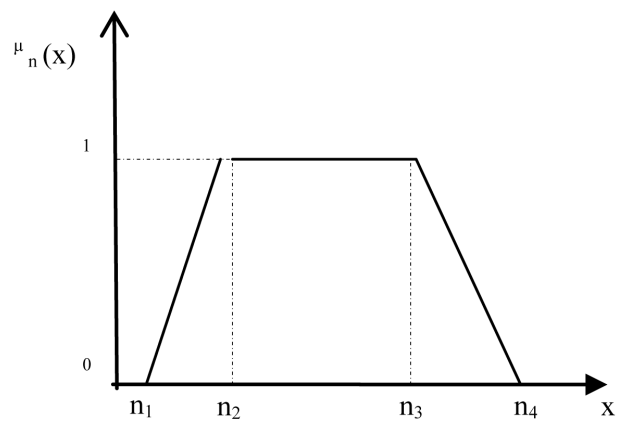

Figure 1. Trapeze fuzzy number. 
If $n_{2}=n_{3}$ in $\bar{n}=\left(n_{1}, n_{2}, n_{3}, n_{4}\right)$ trapeze fuzzy number, then it converts into a $\bar{n}$ triangle fuzzy number.

While using TOPSIS method, some operations on fuzzy numbers must be paid attention to. Let's assume that we are given two trapeze fuzzy numbers $\bar{n}=\left(n_{1}, n_{2}, n_{3}, n_{4}\right)$ and $\bar{m}=\left(m_{1}, m_{2}, m_{3}, m_{4}\right)$.

Following extensibility principal must be met for their fuzzy sum, difference and multiplication:

$$
\begin{aligned}
& \bar{n} \oplus \bar{m}=\left[n_{1}+m_{1}, n_{2}+m_{2}, n_{3}+m_{3}, n_{4}+m_{4}\right], \\
& \bar{n}-\bar{m}=\left[n_{1}-m_{4}, n_{2}-m_{3}, n_{3}-m_{2}, n_{4}-m_{1}\right], \\
& \bar{n} \otimes \bar{m} \cong\left[n_{1} m_{1}, n_{2} m_{2}, n_{3} m_{3}, n_{4} m_{4}\right], \\
& \bar{n} \otimes r=\left[n_{1} r, n_{2} r, n_{3} r, n_{4} r\right] .
\end{aligned}
$$

Definition 2. Let's assume that two $\bar{n}=\left(n_{1}, n_{2}, n_{3}, n_{4}\right)$ and $\bar{m}=\left(m_{1}, m_{2}, m_{3}, m_{4}\right)$ trapeze fuzzy numbers are given. The distance between them is calculated as following [28] [36].

$$
d_{c}(\bar{n}, \bar{m})=\sqrt{\frac{1}{4}\left(\left(n_{1}-m_{1}\right)^{2}+\left(n_{2}-m_{2}\right)^{2}+\left(n_{3}-m_{3}\right)^{2}+\left(n_{4}-m_{4}\right)^{2}\right)}
$$

If $\bar{n}$ and $\bar{m}$ are similar (same) fuzzy numbers, then $d_{c}(\bar{n}, \bar{m})=0$.

In order to apply this method, unit quality measuring scale is accepted [7]-[9], each evaluable criterion indicator is graduated in accordance with 7 level quality evaluation degrees and their trapeze fuzzy number conversion principal is referred to (Figure 2).

Trapeze fuzzy evaluation table of linguistic quality degrees is as following. Based on Table 1, a fuzzy number can be found for each linguistic expression.

For example, the fuzzy number of "medium good" linguistic expression is defined as $(5,6,7,8)$ out of 10 point rating. Then the fuzzification of "medium good" can be demonstrated as following:

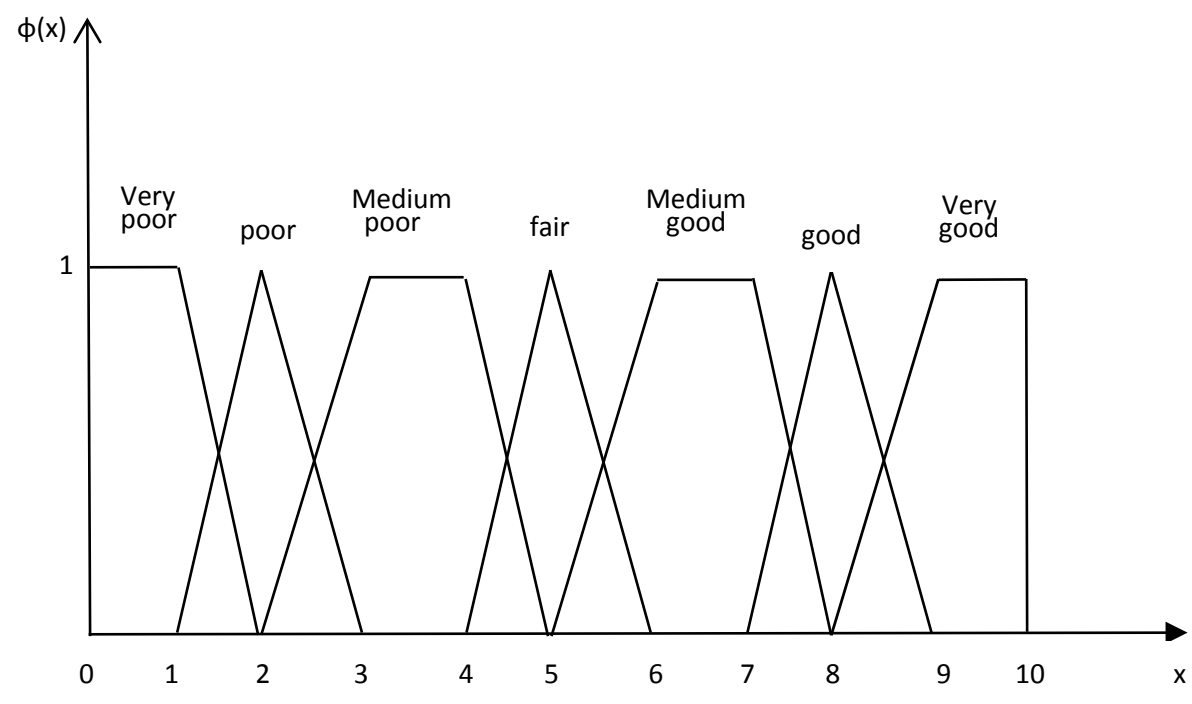

Figure 2. Conversion of linguistic expression to fuzzy number based on rating.

Table 1. Conversion of linguistic expression to fuzzy number based on rating.

\begin{tabular}{cc}
\hline Linguistic expression & Fuzzy number \\
\hline Very poor & $(0,0,1,2)$ \\
Poor & $(1,2,2,3)$ \\
Medium poor & $(2,3,4,5)$ \\
Fair & $(4,5,5,6)$ \\
Medium good & $(5,6,7,8)$ \\
Good & $(7,8,8,9)$ \\
Very good & $(8,9,10,10)$ \\
\hline
\end{tabular}




$$
\varphi_{\text {medium good }}(x)= \begin{cases}0, & x<5, \\ \frac{x-5}{6-5}, & 5 \leq x \leq 6, \\ 1, & 6 \leq x \leq 7, \\ \frac{x-8}{7-8}, & 7 \leq x \leq 8, \\ 1, & x>8 .\end{cases}
$$

\section{TOPSIS Method in Solution of Personnel Selection Problem}

\subsection{Problem Statement}

To provide correctness and objectiveness of decisions made in relation with staff management in the organization, decision options in relevance with each problem statement are defined and evaluation objects—alternatives and characterizing criteria, indicator system defining these criteria is formed, they are evaluated by finding the relevance degree of alternatives to these indicators and depending on this value, decision option related to them (alternatives) is selected. Thus, let's assume that:

1. $X=\left\{x_{i}, i=\overline{1, n}\right\}$-is a set of evaluated alternatives and the best alternative must be chosen, for example, candidates to be hired in the hiring issue;

2. $K=\left\{k_{j}, j=\overline{1, m}\right\}$-is a set of criteria with different weights relevant to criteria (for example criteria characterizing hired people) and these criteria are also defined based on multiple indicators with different weights;

3. $k_{j}=\left\{k_{j t}, t=\overline{1, s_{j}}\right\}$ 一evaluable criteria indicators with different weights;

4. $E=\left\{e_{l}, l=\overline{1, g}\right\}$ — set of experts evaluating the relevance of alternatives to criteria indicators.

Objective: evaluation and regulation of alternatives based on linguistic expressions of quality used by the experts reflecting the relevance of alternatives to criteria indicators with different weights.

\subsection{Solution of the Problem}

Headings, or heads, are organizational devices that guide the reader through your paper. There are two types: component heads and text heads.

$\mathbf{1}^{\text {st }}$ Step. Referring to methods described in [30] [37], importance coefficient $w_{j}\left(\sum_{j=1}^{m} w_{j}=1\right)$ and importance coefficients $w_{j t}, t=\overline{1, s_{j}}, j=\overline{1, m}$ are defined. Later, by referring to hierarchic analysis method, weightweight coefficient of each $k_{j t}, j=\overline{1, m}, t=\overline{1, s}$ criteria indicator in generalizing $K=\left\{k_{j}, j=\overline{1, m}\right\} \quad$ criteria is defined.

$$
w_{z}=w_{j t}^{K}=w_{j t} \cdot w_{j} .
$$

Here:

$$
z=s_{j-1}+t, \quad j=\overline{1, m}, \quad t=\overline{1, s_{j}}, \quad s_{0}=0 \quad \text { and } \quad z=\overline{1, Z} .
$$

$Z$-is the general number of criteria indicators.

$2^{\text {nd }}$ Step. Relevance level of alternatives to criteria indicators are expressed in accordance with seven quality levels of our language (very poor, poor, medium poor, fair, medium good, good, very good). Each such expression is a quality level forming relevance- $\left\{\phi_{k z}\left(x_{i}\right)\right\}$ of $k_{z}$ evaluable criteria indicator of $x_{i}$ alternative, and is expressed in relevant trapeze $R^{l}=\left(r_{i z}^{l}\right)=\left(a_{i z}^{l}, b_{i z}^{l}, c_{i z}^{l}, d_{i z}^{l}\right)$ with a fuzzy number. For example, if relevance of $x_{i}$ alternative to any $k_{z}$ criteria is evaluated by expert $l$ as "good", then, its conversion to a fuzzy number in trapeze is expressed as "good" $r_{i z}^{l}=(7,8,8,9)$, and if evaluated as "very good" is expressed as, "veru good"; 
$r_{i z}^{l}=(8,9,10,10)$.

Linguistic expression of relevance of alternatives to criteria indicators by experts result in $R=\left[r_{i z}^{l}\right]_{i \times z \times l}$ matrix with $i \times z \times l$ dimensions.

$3^{\text {rd }}$ Step. Based on individual evaluation of experts- $E=\left\{e_{l}, l=\overline{1, g}\right\}$, single-generalized matrix referring to $g$ number of matrixes defined by trapeze fuzzy numbers expressing relevance of $x_{i}$ alternative to $k_{z}$ criteria is defined, i.e.:

$$
R^{l}=\left[r_{i z}^{l}\right], \quad l=\overline{1, g} \Leftrightarrow\left\{a_{i z}^{l}, b_{i z}^{l}, c_{i z}^{l}, d_{i z}^{l}\right\}, \quad l=\overline{1, g} \Leftrightarrow R_{i z}=\left[r_{i z}\right] \Leftrightarrow\left\{a_{i z}, b_{i z}, c_{i z}, d_{i z}\right\}
$$

Here:

$$
\begin{aligned}
& a_{i z}=\left\{\min a_{i z}^{l}, l=\overline{1, g}\right\} ; \\
& b_{i z}=\frac{1}{g} \sum_{l=1}^{g} b_{i z}^{l} ; \\
& c_{i z}=\frac{1}{g} \sum_{l=1}^{g} c_{i z}^{l} ; \\
& d_{i z}=\left\{\max d_{i z}^{l}, l=\overline{1, g}\right\} .
\end{aligned}
$$

As a result we obtain a $i \times z$ dimensional $R_{i z}=\left[r_{i z}\right]$ matrix.

$4^{\text {th }}$ Step. $R_{i z}=\left[r_{i z}\right] \Leftrightarrow\left\{a_{i z}, b_{i z}, c_{i z}, d_{i z}\right\}$ fuzzy number matrix is normalized. For this, values with different dimensions in [0,1] interval are converted into fuzzy numbers using Hsu and Chen method [38]. Based on this method, $d_{z}^{+}=\operatorname{maxd}_{i z}, i=\overline{1, n}$ is defined, elements of normalized matrix are defined using following formulas:

$$
R_{i z}^{n}=\left[r_{i z}^{n}\right] \Leftrightarrow\left\{a_{i z}^{n}, b_{i z}^{n}, c_{i z}^{n}, d_{i j z}^{n}\right\} \Leftrightarrow\left\{\frac{a_{i z}}{d_{z}^{+}}, \frac{b_{i z}}{d_{z}^{+}}, \frac{c_{i z}}{d_{z}^{+}}, \frac{d_{i z}}{d_{z}^{+}}\right\} .
$$

$5^{\text {th }}$ Step. All elements of normalized $R_{i z}^{n}=\left[r_{i z}^{n}\right] \Leftrightarrow\left\{a_{i z}^{n}, b_{i z}^{n}, c_{i z}^{n}, d_{i z}^{n}\right\}$ matrix are multiplied by weights of criteria indicators. For this, $\bar{n} \otimes r \cong\left[n_{1} r, n_{2} r, n_{3} r, n_{4} r\right]$ phrase from condition (1) is used. Let's define fuzzy number matrix by consideration of weight coefficients of criteria indicators: $\bar{R}_{i z}=\left[\bar{r}_{i z}\right] \Leftrightarrow\left\{\bar{a}_{i z}, \bar{b}_{i z}, \bar{c}_{i z}, \bar{d}_{i z}\right\}$.

Here:

$$
\begin{aligned}
& \overline{a_{i z}}=a_{i j} \cdot w_{z} ; \\
& \overline{b_{i z}}=b_{i j} \cdot w_{z} ; \\
& \overline{c_{i z}}=c_{i j} \cdot w_{z} ; \\
& \overline{d_{i z}}=d_{i j} \cdot w_{z} .
\end{aligned}
$$

$6^{\text {th }}$ Step. On grounds of existing alternatives, trapeze fuzzy numbers of $X^{*}$-ideal solution option (ISO) in accordance with each criteria indicator is calculated. For this, each $d_{p}^{*}=\left\{\max \overline{d_{i z}}, i=\overline{1, n}\right\}$ is selected based on $\bar{r}=\left\{\overline{a_{i z}}, \overline{b_{i z}}, \overline{c_{i z}}, \overline{d_{i z}}\right\}$ fuzzy number in accordance with $k_{z}, z=\overline{1, Z}$ criteria indicator of each $x_{i}$ alternative and as a result, following single matrix based on fuzzy number relevant to criteria indicators of ideal solution option is determined:

$$
X^{*}=\left[d_{z}^{*}\right]=\left[\left(d_{1}^{*}, d_{1}^{*}, d_{1}^{*}, d_{1}^{*}\right), \cdots,\left(d_{z}^{*}, d_{Z}^{*}, d_{Z}^{*}, d_{Z}^{*}\right)\right] .
$$

$7^{\text {th }}$ Step. On grounds of existing alternatives, trapeze fuzzy numbers of $X^{-}$-extremely bad solution (EBS) in accordance with each criteria indicator is calculated. For this, $a_{p}^{-}=\left\{\min \overline{a_{i z}}, i=\overline{1, n}\right\}$ based on $\overline{r_{i z}}=\left(\overline{a_{i z}}, \overline{b_{i z}}, \overline{c_{i z}}, \overline{d_{i z}}\right)$ -fuzzy number in accordance with $k_{z}, z=\overline{1, Z}$ criteria indicator of each $x_{i}$ alternative is found and following single matrix is developed: 


$$
X^{-}=\left[a_{Z}^{-}\right]=\left[\left(a_{1}^{-}, a_{1}^{-}, a_{1}^{-}, a_{1}^{-}\right), \cdots,\left(a_{Z}^{-}, a_{Z}^{-}, a_{Z}^{-}, a_{Z}^{-}\right)\right] .
$$

$\mathbf{8}^{\text {th }}$ Step. At this stage, fuzzy number matrix reflecting proximity of alternatives to ideal solution option is developed.

$x_{i}=\overline{r_{i z}}=\left(\overline{a_{i z}}, \overline{b_{i z}}, \overline{c_{i z}}, \overline{d_{i z}}\right)$ of each $x_{i}$ alternative is defined based on fuzzy number (2) formula reflecting proximity of any $k_{j t}$ criteria indicator to ISO as following:

$$
D_{z}^{*}\left(x_{i}, X^{*}\right)=\sqrt{\frac{1}{4}\left(\left(\overline{a_{i z}}-d_{z}^{*}\right)^{2}+\left(\overline{b_{i z}}-d_{z}^{*}\right)^{2}+\left(\overline{c_{i z}}-d_{z}^{*}\right)^{2}+\left(\overline{d_{i z}}-d_{z}^{*}\right)^{2}\right)}
$$

$\left[D^{*}\right]$ ISO proximity matrix with $i \times j$ dimensions reflecting obtained results is developed.

$\mathbf{9}^{\text {th }}$ Step. Fuzzy number reflecting remoteness of alternatives to EBS is found.

$$
D_{z}^{-}\left(x_{i}, X^{-}\right)=\sqrt{\frac{1}{4}\left(\left(\overline{a_{i z}}-a_{z}^{-}\right)^{2}+\left(\overline{b_{i z}}-a_{z}^{-}\right)^{2}+\left(\overline{c_{i z}}-a_{z}^{-}\right)^{2}+\left(\overline{d_{i z}}-a_{z}^{-}\right)^{2}\right)}
$$

$\left[D^{-}\right]$EBS remoteness matrix with $i \times j$ dimensions reflecting obtained results is developed.

$10^{\text {th }}$ Step. Proximity of each alternative of all criteria to ISO is calculated with following formula:

$$
D^{*}\left(x_{i}\right)=\sqrt{\sum_{z=1}^{Z}\left(D_{z}^{*}\left(x_{i}, X^{*}\right)\right)^{2}}
$$

$\mathbf{1 1}^{\text {th }}$ Step. Remoteness of each alternative from EPS in accordance with all criteria is calculated with following formula:

$$
D^{-}\left(x_{i}\right)=\sqrt{\sum_{z=1}^{Z}\left(D_{z}^{-}\left(x_{i}, X^{*}\right)\right)^{2}}
$$

$12^{\text {th }}$ Step. Based on values of proximity of alternatives to ISO and their remoteness from EBS, numerical value of their relevance to ideal solution is calculated and normalized.

$$
D\left(x_{i}\right)=D^{*}\left(x_{i}\right)+D^{-}\left(x_{i}\right), \quad \varphi_{K}\left(x_{i}\right)=\frac{D^{-}\left(x_{i}\right)}{D\left(x_{i}\right)} .
$$

Regulated order of obtained results from maximum to minimum (or vice versa) is relevant to regulated order of alternatives from good to bad (or vice versa).

\section{Application of TOPSIS Method for Decision-Making in Personnel Selection Problem}

Referring to fuzzy logic formalism, fuzzy TOPSIS method was used for evaluation and selection of alternatives in realization of decision making support system in hiring issues of candidates. For realization of the system, primarily a general criteria system is formed in order to evaluate hired employees to the plant. This system contains criteria and characterizing indicators allowing evaluating candidates hiring to any department or position at the plant.

Candidate evaluation issue for hiring to HRM department of the plant has been reviewed during conducted experiment. For this purpose, following criteria and criteria indicators were determined from the general criteria system with participation of experts for appointment to the position:

$K_{1}$-science and education criteria and indicators characterizing it:

$k_{11}$-relevance of completed education to corresponding job;

$k_{12}$ - character of investigator.

$K_{2}$-behavior and appearance criteria and indicators characterizing it:

$k_{21}$-balanced;

$k_{22}$-well-conducted and polite.

$K_{3}$-personal psychological criteria and indicators characterizing it: 
$k_{31}$-hardworking, industrious;

$k_{32}$-creative;

$k_{33}$-loyal;

$k_{34}$-high intelligence.

$K_{4}$-functional activity criteria and indicators characterizing it:

$k_{41}$-work capability;

$k_{42}$-learning capability.

$K_{5}$-medical criteria and indicators characterizing it:

$k_{51}$-physical health;

$k_{52}$-spiritual and psychological health.

Results obtained from evaluation of these indicators-will define the value of chance- $K$-hiring chance of the candidate.

Result to be obtained based on fuzzy TOPSIS method- $\varphi_{K}\left(x_{i}\right)$, will express the hiring chance of $x_{i}$ candidate as a value defined in $[0,1]$ interval. Depending on this value, experts pre-form following hiring decision options:

1. If $\varphi_{K}\left(x_{i}\right) \in[0,0.25)$, then this candidate decidedly cannot be hired;

2. If $\varphi_{K}\left(x_{i}\right) \in[0.25,0.45)$, hiring of this candidate carries great risk;

3. If $\varphi_{K}\left(x_{i}\right) \in[0.45,0.62)$, hiring of this candidate carries a bit of risk;

4. If $\varphi_{K}\left(x_{i}\right) \in[0.62,0.8)$, this candidate can be hired;

5. If $\varphi_{K}\left(x_{i}\right) \in[0.8,1]$, this candidate is unconditionally hired.

In the next stage, importance coefficients of these criteria and their characterizing indicators relatively to each other are defined, for this objective paired comparison method is referred to, detection of contradictions in experts' evaluation is reviewed [30] [37]. Based on obtained results, weight coefficients of criteria indicators have been defined in accordance with hierarchic analysis method (Table 2).

Relevance of hiring of 3 candidates to listed criteria indicators has been evaluated in accordance with Table 3 with participation of 4 experts.

Based on Formula (3), single trapeze matrix is developed based on individual evaluation of experts. Results of single trapeze fuzzy matrix in accordance with $k_{11}$ and $k_{12}$ criteria indicators are provided below (Table 4).

Table 2. Weight coefficient of criteria indicators.

\begin{tabular}{|c|c|c|c|c|}
\hline Criteria & $\begin{array}{c}\text { Importance } \\
\text { coefficients of criteria }\end{array}$ & Criteria indicator & $\begin{array}{l}\text { Importance coefficients } \\
\text { of criteria indicators }\end{array}$ & $\begin{array}{l}\text { Weight coefficients } \\
\text { of criteria indicators }\end{array}$ \\
\hline \multirow{2}{*}{$K_{1}$} & \multirow{2}{*}{0.11} & $k_{11}$ & 0.54 & 0.06 \\
\hline & & $k_{12}$ & 0.46 & 0.05 \\
\hline \multirow{2}{*}{$K_{2}$} & \multirow{2}{*}{0.08} & $k_{21}$ & 0.47 & 0.04 \\
\hline & & $k_{22}$ & 0.53 & 0.04 \\
\hline \multirow{4}{*}{$K_{3}$} & \multirow{4}{*}{0.4} & $k_{31}$ & 0.2 & 0.08 \\
\hline & & $k_{32}$ & 0.22 & 0.13 \\
\hline & & $k_{33}$ & 0.26 & 0.10 \\
\hline & & $k_{34}$ & 0.32 & 0.09 \\
\hline \multirow{2}{*}{$K_{4}$} & \multirow{2}{*}{0.1} & $k_{41}$ & 0.63 & 0.06 \\
\hline & & $k_{42}$ & 0.37 & 0.04 \\
\hline \multirow{2}{*}{$K_{5}$} & \multirow{2}{*}{0.31} & $k_{51}$ & 0.35 & 0.11 \\
\hline & & $k_{52}$ & 0.65 & 0.20 \\
\hline
\end{tabular}


Table 3. Evaluation of criteria indicators.

\begin{tabular}{|c|c|c|c|c|c|}
\hline Criteria indicators & Alternatives & Expert 1 & Expert 2 & Expert 3 & Expert 4 \\
\hline & $x_{1}$ & good & good & good & good \\
\hline \multirow[t]{3}{*}{$k_{11}$} & $x_{2}$ & good & very good & fair & medium good \\
\hline & $x_{3}$ & good & good & very good & good \\
\hline & $x_{1}$ & very good & very good & good & very good \\
\hline \multirow[t]{3}{*}{$k_{12}$} & $x_{2}$ & medium good & good & very good & medium good \\
\hline & $x_{3}$ & good & very good & very good & medium \\
\hline & $x_{1}$ & good & good & fair & medium good \\
\hline \multirow[t]{3}{*}{$k_{21}$} & $x_{2}$ & good & very good & very good & good \\
\hline & $x_{3}$ & very good & good & good & very good \\
\hline & $x_{1}$ & medium good & good & very good & good \\
\hline \multirow[t]{3}{*}{$k_{22}$} & $x_{2}$ & very good & very good & very good & very good \\
\hline & $x_{3}$ & good & very good & good & good \\
\hline & $x_{1}$ & good & medium good & very good & good \\
\hline \multirow[t]{3}{*}{$k_{31}$} & $x_{2}$ & very good & good & good & very good \\
\hline & $x_{3}$ & good & very good & very good & good \\
\hline & $x_{1}$ & good & good & very good & good \\
\hline \multirow[t]{3}{*}{$k_{32}$} & $x_{2}$ & very good & medium good & good & very good \\
\hline & $x_{3}$ & medium good & good & very good & medium good \\
\hline & $x_{1}$ & very good & very good & very good & very good \\
\hline \multirow[t]{3}{*}{$k_{33}$} & $x_{2}$ & good & very good & good & good \\
\hline & $x_{3}$ & good & good & fair & medium good \\
\hline & $x_{1}$ & good & very good & very good & fair \\
\hline \multirow[t]{3}{*}{$k_{34}$} & $x_{2}$ & good & very good & good & good \\
\hline & $x_{3}$ & medium good & good & very good & good \\
\hline & $x_{1}$ & very good & good & good & very good \\
\hline \multirow[t]{3}{*}{$k_{41}$} & $x_{2}$ & good & good & very good & good \\
\hline & $x_{3}$ & very good & medium good & good & very good \\
\hline & $x_{1}$ & medium good & good & very good & medium good \\
\hline \multirow[t]{3}{*}{$k_{42}$} & $x_{2}$ & good & very good & very good & fair \\
\hline & $x_{3}$ & good & very good & Good & good \\
\hline & $x_{1}$ & very good & good & Good & good \\
\hline \multirow[t]{3}{*}{$k_{51}$} & $x_{2}$ & medium good & good & very good & good \\
\hline & $x_{3}$ & very good & very good & very good & very good \\
\hline & $x_{1}$ & good & very good & Good & good \\
\hline \multirow[t]{2}{*}{$k_{52}$} & $x_{2}$ & good & good & Fair & medium good \\
\hline & $x_{3}$ & good & very good & very good & good \\
\hline
\end{tabular}


Table 4. Single trapeze fuzzy matrix in accordance with $k_{11}$ and $k_{12}$ criteria indicators.

\begin{tabular}{ccccccc}
\hline $\begin{array}{c}\text { Criteria } \\
\text { indicators }\end{array}$ & Alternatives & Expert 1 & Expert 2 & Expert 3 & Expert 4 & $\begin{array}{c}\text { Generalized single } \\
\text { trapeze fuzzy number }\end{array}$ \\
\hline$k_{11}$ & $x_{1}$ & $(7,8,8,9)$ & $(7,8,8,9)$ & $(7,8,8,9)$ & $(7,8,8,9)$ & $(7,8,8,9)$ \\
& $x_{2}$ & $(7,8,8,9)$ & $(8,9,10,10)$ & $(4,5,5,6)$ & $(5,6,7,8)$ & $(4,7,7.5,10)$ \\
& $x_{3}$ & $(7,8,8,9)$ & $(7,8,8,9)$ & $(8,9,10,10)$ & $(7,8,8,9)$ & $(7,8.3,9,10)$ \\
& $x_{1}$ & $(8,9,10,10)$ & $(8,9,10,10)$ & $(7,8,8,9)$ & $(8,9,10,10)$ & $(7,8.8,9.5,10)$ \\
$k_{12}$ & $x_{2}$ & $(5,6,7,8)$ & $(7,8,8,9)$ & $(8,9,10,10)$ & $(5,6,7,8)$ & $(5,7.3,8,10)$ \\
& $x_{3}$ & $(7,8,8,9)$ & $(8,9,10,10)$ & $(8,9,10,10)$ & $(4,5,5,6)$ & $(4,7.8,8.3,10)$ \\
\hline
\end{tabular}

Based on (4) formula, single trapeze matrix is formed and all its elements are multiplied by weight coefficients of criteria in accordance with Formula (5) and resultants are given in Table 5.

ISO and EBS single matrixes are developed on existing grounds of alternatives. ISO proximity matrix $\left[D^{*}\right]$ with $i \times j$ dimensions reflecting the obtained results is given in Table 6 .

EBS remoteness proximity $\left[D^{-}\right]$with $i \times j$ dimensions reflecting remoteness of alternatives from EBS is as following and resultants are given in Table 7.

Numerical value of proximity to ISO, remoteness from EXP and relevance to ideal solution of each alternative in accordance with all criteria is calculated resultants are given in Table $\mathbf{8}$.

Based on obtained results, the best solution option is $x_{3}$ alternative and the value of its hiring chance is $\varphi_{K}\left(x_{3}\right)=0.63$. In accordance with decision options of the experts: this candidate can be hired. In accordance with next listing, $x_{2}$ alternative is $\varphi_{K}\left(x_{2}\right)=0.58$. And $x_{1}$ alternatives equal to $\varphi_{K}\left(x_{1}\right)=0.55$ and their hiring chance value matches the identical decision option: hiring of this candidate can carry a bit of risk.

\section{Results}

The proposed approach was foreseen for realization of support system applied to decision-making issues in the employment of applicants within SOCAREF2013 ${ }^{1}$ grant project. It should be noted that at the moment SOCAR department of human resourses management for applicants evaluation in the enployment issues realization bases on their precise report indexes, expert assessment defined on the bases of quality competition and final results are determined by point system. In order to ground benefits of the approach proposed in the article the results of approach application have been compared with the results of point system application herewith.

With this purpose let's look through alternatives decision formation given in Table 3 that was compiled on the basis of expert evaluation with references to point system.

For alternatives evaluation on the basis of points system "very good" equals 10 points, "good" -8 points, "medium good" -6 points and "fair" -4 points.

Table 9 given below is being compiled on the basis of statistic results of evaluation by considered three alternatives criteria indexes according to Table 3.

According to the statistic results obtained on the basis of point system the correspondence degree of each alternative to the ideal solution (480 points) is defined. In this case the highest point alternative $x_{3}$ is very good, next is $x_{1}$ and the last is $x_{2}$ alternative.

The decision per each alternative is made according to its ideal sulution correspondence estimate:

$x_{1}$ alternative $\left(\varphi_{K}\left(x_{1}\right)=0.833\right)$-this candidate is unconditionally hired.

$x_{2}$ alternative $\left(\varphi_{K}\left(x_{2}\right)=0.825\right)$-this candidate is unconditionally hired.

$x_{3}$ alternative $\left(\varphi_{K}\left(x_{3}\right)=0.846\right)$ - this candidate is unconditionally hired.

The results obtained according to the proposed method are compared with the results of application of point system approach in Table 10 herein:

${ }^{1}$ SOCAREF2013-Development of problem-solving mechanism of human resources efficient management by application of intelligent information technologies for SOCAR. 
Table 5. Normalized fuzzy number of relevance of alternatives to indicators of criteria.

\begin{tabular}{ccccc}
\hline Criteria indicators & Alternatives & $\begin{array}{c}\text { Normalized single } \\
\text { trapeze fuzzy number }\end{array}$ & $\begin{array}{c}\text { Weight coefficients } \\
\text { of } k_{j t}\end{array}$ & $\begin{array}{c}\text { Normalized fuzzy number } \\
\text { of relevance of alternatives }\end{array}$ \\
\hline$k_{11}$ & $x_{1}$ & $(0.7,0.8,0.8,0.9)$ & & $0.042,0.048,0.048,0.054$ \\
& $x_{2}$ & $(0.4,0.7,0.75,1)$ & 0.06 & $0.024,0.042,0.045,0.06$ \\
$x_{3}$ & $(0.7,0.83,0.9,1)$ & & $0.042,0.05,0.054,0.06$ \\
$k_{12}$ & $x_{1}$ & $(0.7,0.88,0.95,1)$ & 0.05 & $0.035,0.044,0.098,0.05$ \\
& $x_{2}$ & $(0.5,0.73,0.8,1)$ & & $0.025,0.037,0.04,0.05$ \\
$x_{3}$ & $(0.4,0.78,0.83,1)$ & & $0.02,0.039,0.042,0.05$ \\
\hline
\end{tabular}

Table 6. ISO proximity matrix $\left[D^{*}\right]$.

\begin{tabular}{cccc}
\hline Criteria indicators & $D\left(x_{1} X^{*}\right)$ & $D\left(x_{2} X^{*}\right)$ & $D\left(x_{3} X^{*}\right)$ \\
\hline$k_{11}$ & 0.45 & 0.51 & 0.4 \\
$k_{12}$ & 0.34 & 0.49 & 0.49 \\
$k_{21}$ & 0.57 & 0.36 & 0.4 \\
$k_{22}$ & 0.67 & 0.27 & 0.35 \\
$k_{31}$ & 0.48 & 0.37 & 0.37 \\
$k_{32}$ & 0.56 & 0.49 & 0.43 \\
$k_{33}$ & 0.37 & 0.4 & 0.47 \\
$k_{34}$ & 0.5 & 0.39 & 0.37 \\
$k_{41}$ & 0.37 & 0.39 & 0.29 \\
$k_{42}$ & 0.49 & 0.49 & 0.39 \\
$k_{51}$ & 0.39 & 0.47 & 0.27 \\
$k_{52}$ & 0.4 & 0.6 & 0.33 \\
\hline
\end{tabular}

Table 7. EBS remoteness proximity $\left[D^{*}\right]$.

\begin{tabular}{cccc}
\hline Criteria indicators & $D\left(x_{1} X^{-}\right)$ & $D\left(x_{2} X^{-}\right)$ & $D\left(x_{3} X^{-}\right)$ \\
\hline$k_{11}$ & 0.55 & 0.56 & 0.68 \\
$k_{12}$ & 0.7 & 0.6 & 0.6 \\
$k_{21}$ & 0.52 & 0.68 & 0.66 \\
$k_{22}$ & 0.53 & 0.65 & 0.71 \\
$k_{31}$ & 0.52 & 0.51 & 0.6 \\
$k_{32}$ & 0.52 & 0.53 & 0.67 \\
$k_{33}$ & 0.63 & 0.66 & 0.62 \\
$k_{34}$ & 0.59 & 0.67 & 0.62 \\
$k_{41}$ & 0.51 & 0.59 & 0.64 \\
$k_{42}$ & 0.6 & 0.6 & 0.67 \\
$k_{51}$ & 0.59 & 0.52 & 0.51 \\
$k_{52}$ & 0.74 & 0.51 & 0.64 \\
\hline
\end{tabular}


Table 8. Normalized relevance to ideal solution of each alternative.

\begin{tabular}{ccccc}
\hline & $X^{*}$ & $X^{-}$ & $X^{*}+X^{-}$ & $\varphi_{K}\left(x_{i}\right)$ \\
\hline$D\left(x_{1} X^{-}\right)$ & 5.59 & 7.02 & 12.61 & 0.55 \\
$D\left(x_{2} X^{-}\right)$ & 5.23 & 7.08 & 12.31 & 0.58 \\
$D\left(x_{3} X^{-}\right)$ & 4.56 & 7.76 & 12.32 & 0.63 \\
\hline
\end{tabular}

Table 9. Final results according to point system of alternatives.

\begin{tabular}{ccccccc}
\hline Alternatives & Very good & Good & Medium good & Fair & Sum & Correspondence of alternatives to ideal solution \\
\hline$x_{1}$ & 17 & 24 & 5 & 2 & 400 & 0.833 \\
$x_{2}$ & 18 & 21 & 6 & 3 & 396 & 0.825 \\
$x_{3}$ & 19 & 22 & 6 & 1 & 406 & 0.846 \\
\hline
\end{tabular}

Table 10. Comparision of the results.

\begin{tabular}{ccc}
\hline Alternatives & \multicolumn{1}{c}{ FDMSM } & Results of point system \\
\hline$x_{1}$ & Hiring of this candidate can carry a bit of risk. & This candidate is unconditionally hired. \\
$x_{2}$ & Hiring of this candidate can carry a bit of risk. & This candidate is unconditionally hired. \\
$x_{3}$ & This candidate can be hired. & This candidate is unconditionally hired. \\
\hline
\end{tabular}

As it is seen the results of point system application in the solution of applicant employment problem differs from the results of proposed FDMSM application and this method supports the employment of "unconditionally hired" employee rather than "risk-carrying" as appointed by point system.

\section{Conclusions}

Traditional methods of multi-criteria assessment and ordering cannot effectively solve the problem of the group (collective) decision making under imprecise and linguistic information. Basing on the proposed model and method, the key features of more objective and transparent management decisions of the personnel are as follows:

- The number of criteria and criteria indicators characterizing the issue are not restricted;

- The quality of criteria indicators characterizing the issue, the importance and advantage of criteria and criteria indicators in relation to each other are taken into account, and the conflict is determined;

- Subjectivity of decision-maker in the decision-making process are reduced, more objective and transparent decisions are made.

The proposed method for multi-criteria assessment and ranking can be applied for solving the problems of Personnel Management, as well as for other problems arising from the human activity.

However, the criteria, assessment indicators characterizing assessed objects should be formed previously and their importance coefficients should be determined using appropriate methods. Quite difficult and time-consuming procedure for implementing the above steps is a weak point of the proposed approach.

One of the advantages of this approach is possibility of taking into consideration the competence of the participating experts in appropriate subject domain. Thus, Decision-Maker do not always consider these experts' competency equal in appropriate subject and their competence is considered only within decision-making process. That's why we are planning to consider this parameter also in our future researches.

\section{References}

[1] Cole, G.A. (2002) Personnel and Human Resource Management. 5th Edition, Thomson Learning, Wadsworth, 448 p.

[2] Spencer, M.L. and Spencer, S.M. (1993) Competence at Work: Models for Superior Performance. John Wiley \& Sons, 
Inc., New York, 384 p.

[3] Bazarov, T.Y. (2009) Personnel Management. UNITY-DANA Publ., Moscow, 240 p.

[4] Makarova, I.K. (2007) Human Resource Management. DELO Publ., Moscow, 232 p.

[5] Ivantsevich, J.M. and Lobanov, A.A. (2004) Human Resources Management. Gordarike publ., Moscow, 245 p.

[6] Trachtengertz, E.A. (2001) Capabilities and Realization of Computer Decision Making Support Systems. News of Academy of Sciences of Russia. Management Theory and Systems, 3, 86-113.

[7] Larichev, O.I. (2000) Theory and Methods of Decision Making, Logos, Moscow, 296 p.

[8] Mikoni, S.V. (2009) Multicriteria Selection on the Final Alternative Set. Student Handbook, LAN Publ., Saint Petersburg, 270 p.

[9] Mammadova, M.G. and Jabrayilova, Z.Q. (2013) Application of TOPSIS Method in Support of Decisions Made in Staff Management Issues. Computer Technology and Application, 4, 307-316.

[10] Management of Organization Personnel (2005) Manual. Under Edition of Kibanov, Infra-M Publ., Moscow, 638 p. http://fptl.ru/files/menedjment/kibanov ypravlenie-personalom.pdf

[11] Nussbaum, M., Singer, M., Rosas, R., Castillo, M., Flies, E., Lara, R. and Sommers, R. (1999) Decision Support System for Conflict Diagnosis in Personnel Selection. Information \& Management, 36, 55-62. http://dx.doi.org/10.1016/S0378-7206(99)00007-5

[12] Storey Hooper, R., Galvin, T.P., Kilmer, R.A. and Liebowitz, J. (1998) Use of an Expert System in a Personnel Selection Process. Expert Systems with Applications, 14, 425-432. http://dx.doi.org/10.1016/S0957-4174(98)00002-5

[13] Akhlagh, E. (2011) A Rough-Set Based Approach to Design an Expert System for Personnel Selection. World Academy of Science, Engineering and Technology, 54, 202-205.

http://waset.org/Publications/a-rough-set-based-approach-to-design-an-expert-system-for-personnel-selection/14092

[14] Borman, W.C., Hanson, M.A. and Hedge, J.W. (1997) Personnel Selection. Annual Review of Psychology, 48, $299-337$. http://dx.doi.org/10.1146/annurev.psych.48.1.299

[15] Robertson, T. and Smith, M. (2001) Personnel Selection. Journal of Occupational and Organizational Psychology, 74, 441-472. http://dx.doi.org/10.1348/096317901167479

[16] Dursun, M. and Karsak, E. (2010) A Fuzzy MCDM Approach for Personnel Selection. Expert Systems with Applications, 37, 4324-4330. http://dx.doi.org/10.1016/j.eswa.2009.11.067

[17] Chen, L.S. and Cheng, C.H. (2005) Selecting IS Personnel Use Fuzzy GDSS Based on Metric Distance Method. European Journal of Operational Research, 160, 803-820.

[18] Gungor, Z., Serhadlioglu, G. and Kesen, S.E. (2009) A Fuzzy AHP Approach to Personnel Selection Problem. Applied Soft Computing, 9, 641-649. http://dx.doi.org/10.1016/j.asoc.2008.09.003

[19] Wang, Y.J. and Lee, H.S. (2007) Generalizing TOPSIS for Fuzzy Multiple-Criteria Group Decision-Making. Computers and Mathematics with Applications, 53, 1762-1772. http://dx.doi.org/10.1016/j.camwa.2006.08.037

[20] Kelemenis, A. and Askounis, D. (2010) A New TOPSIS-Based Multi-Criteria Approach to Personnel Selections. Expert Systems with Applications, 37, 4999-5008. http://dx.doi.org/10.1016/j.eswa.2009.12.013

[21] Nobari, S. (2011) Design of Fuzzy Decision Support System in Employee Recruitment. Journal of Basic and Applied Scientific Research, 1, 1891-1903.

[22] Mammadova, M.H., Jabrayilova, Z.G. and Nobari, S.M. (2012) Application of TOPSIS Method in Support of Decisions Made in Staff Management Issues. IV International Conference "Problems of Cybernetics and Imformaics" (PCI-2012), Vol. IV, 12-14 September 2012, Baku, 195-198.

[23] Chien, C.F. and Chen, L.F. (2008) Data Mining to Improve Personnel Selection and Enhance Human Capital: A Case Study in High-Technology Industry. Expert System with Applications, 34, 280-290. http://dx.doi.org/10.1016/j.eswa.2006.09.003

[24] Chen, P.C. (2009) A Fuzzy Multiple Criteria Decision Making Model in Employee Recruitment. IJCSNS International Journal of Computer Science and Network Security, 9, 113-117.

[25] Mehrabad, M.S. and Brojeny, M.F. (2007) The Development of on Expert System for Effective Selection and Appointment of the Jobs Applicants in Human Resource Management. Computer and Industrial Engineering, 53, 306-312. http://dx.doi.org/10.1016/j.cie.2007.06.023

[26] Larichev, O.I. and Sternin, M. (1998) Decision Support System of Multi-Objective Problem of Assignment. Information Systems and Processes, 3, 10-16.

[27] Werner, J.M. (2000) Implications of OCB and Contextual Performance for Human Resource Management. Human Resource Management Review, 10, 3-24. http://dx.doi.org/10.1016/S1053-4822(99)00036-4 
[28] Chen, C.T., Lin, C.T. and Huang, S.F. (2006) A Fuzzy Approach for Supplier Evaluation and Selection in Supply Chain Management. International Journal of Production Economics, 102, 289-301. http://dx.doi.org/10.1016/j.ijpe.2005.03.009

[29] Tai, W.S. and Hsu, C.C. (2006) A Realistic Personnel Selection Tool Based on Fuzzy Data Mining Method. Proceedings of the 9th Joint Conference on Information Sciences (JCIS), Taiwan, 8-11 October 2006. file:///C:/Users/HP/Downloads/JCIS06-FTT-56\%20(3).pdf

[30] Saaty, T.L. (1990) How to Make a Decision: The Analytic Hierarchy Process. European Journal of Operational Research, 48, 426-447. http://dx.doi.org/10.1016/0377-2217(90)90057-I

[31] Neumann, J.V. and Morgenstern, O. (2007) Theory of Games and Economic Behavior. One of Princeton University Presses, Notable Centenary Titles, $776 \mathrm{p}$.

[32] Mammadova, M.H., Jabrayilova, Z.G. and Manafli, M.I. (2009) Monitoring of Demands for Information Technology Specialists. Information Technology Publ., Baku, 199 p.

[33] Zadeh, L.A. (1965) Fuzzy Sets. Information and Control, 8, 338-353. http://dx.doi.org/10.1016/S0019-9958(65)90241-X

[34] Kofman, A. (1982) Introduction into the Theory of Fuzzy Sets. Radio and connection, Moscow, 432 p.

[35] Orlovskiy, S.A. (1981) Problems of Decision Making at Fuzzy Initial Information. Nauka, Moscow, 208 p.

[36] Chen, C.T. (2000) Extensions of the TOPSIS for Group Decision-Making under Fuzzy Environment. Fuzzy Sets and Systems, 114, 1-9. http://dx.doi.org/10.1016/S0165-0114(97)00377-1

[37] Jabrailova, Z.G. and Nobari S.M. (2011) Defining Methods of Importance Factor of the Criteria in the Solution of Personnel Management Problems and Detection of Contradictions. Proceedings of the 11th International Conference on Pattern Recognition and Information Processing (PRIP'2011), May 2011, Minsk, 330-333.

[38] Hsu, H.M. and Chen, C.T. (1997) Fuzzy Credibility Relation Method for Multiple Criteria Decision-Making Problems. Information Sciences, 96, 79-91. 
Scientific Research Publishing (SCIRP) is one of the largest Open Access journal publishers. It is currently publishing more than 200 open access, online, peer-reviewed journals covering a wide range of academic disciplines. SCIRP serves the worldwide academic communities and contributes to the progress and application of science with its publication.

Other selected journals from SCIRP are listed as below. Submit your manuscript to us via either submit@scirp.org or Online Submission Portal.
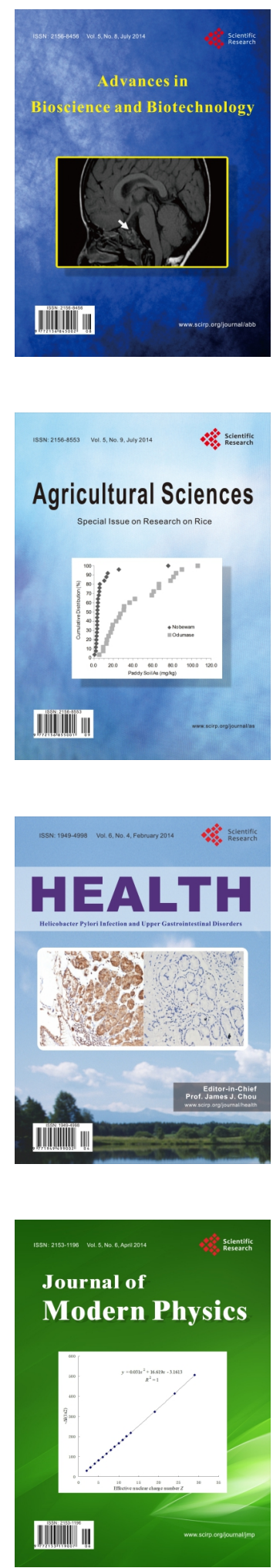
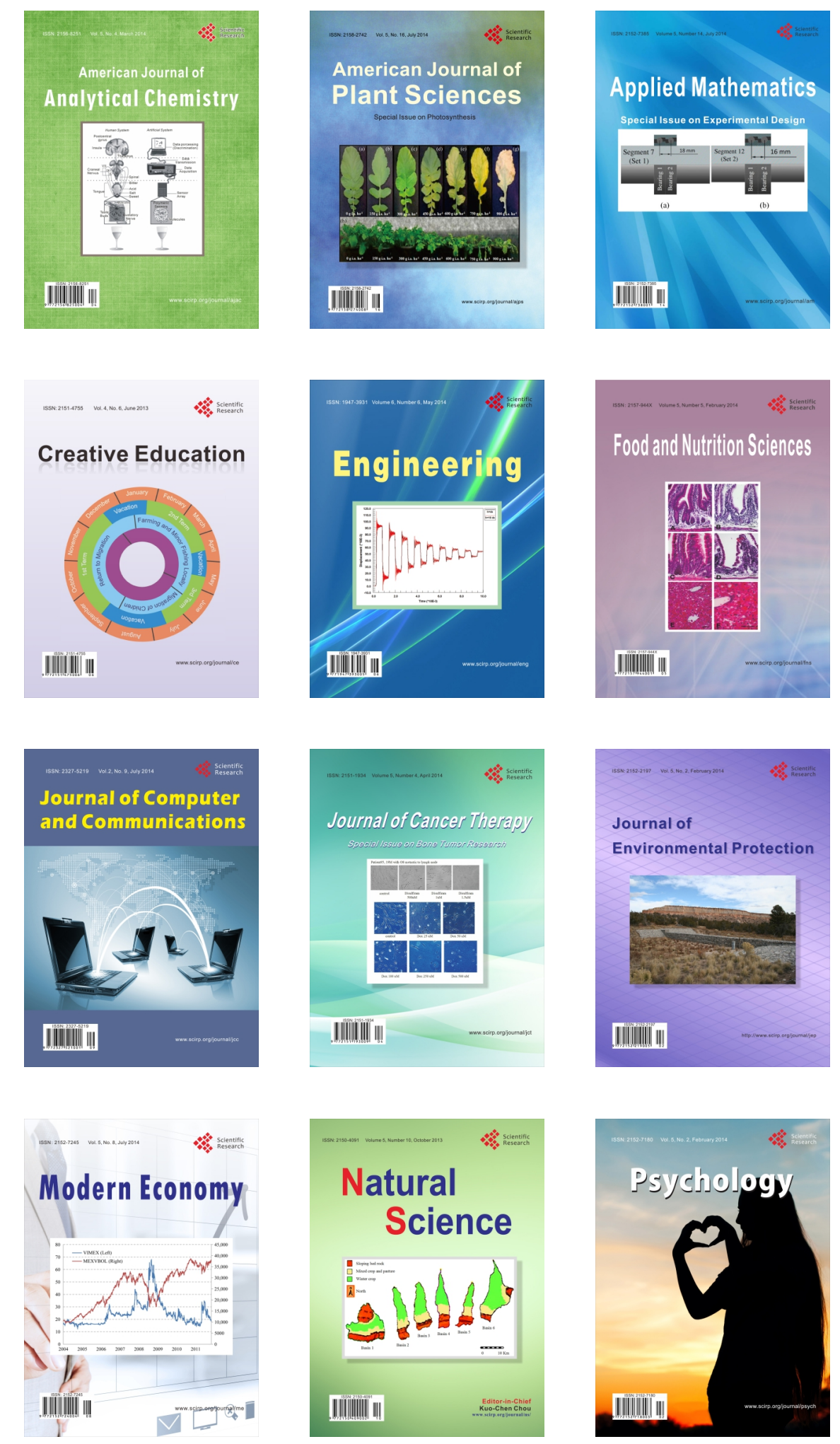